\title{
Mathematica und Internet in der Lehre Betriebswirtschaftliche Modellsammlung PROMA
}

\author{
Matthias Forster, Martin Grabow
}

\section{Einleitung}

Die meisten Studenten der Betriebswirtschaftslehre und der Wirtschaftsinformatik unterschätzen die Bedeutung der Mathematik fuir ihr Studium. Zudem bringen viele Studenten von der Schule eine ablehnende Grundhaltung gegenüber quantitativen Fächern mit. Dies überrascht, da auch vielen Studenten aufgefallen sein wird, daß es einen aktuellen Trend zur Erhöhung des Anteiles mathematischer Modellierung in der Betriebswirtschaftslehre gibt und wegen zunehmender Komplexität auch geben muß.

Unser PROMA-Projekt soll diesem Mangel entgegenwirken, andererseits Interesse wecken und anregen. Die dazu erforderliche Motivation kann nur durch die Kombination geeigneter Mittel verbessert werden. Unser Hauptansatzpunkt dazu ist die eigenständige und aktionsbetonte Erarbeitung von Lerninhalten iiber Beispiele fuir Modelle und Berechnungen. Die Behandlung realistischer Beispiele erforderte noch vor wenigen Jahren einen Rechenaufwand jenseits der Möglichkeiten von Taschenrechner, Bleistift und Papier. Es waren umfangreiche Programmierkenntnisse erforderlich, wenn der Rechner bei der Lösung mathematischer Aufgaben eingesetzt werden sollte.

Heute existiert leistungsfähige, ausgereifte Computeralgebra-Software, mit der sich quantitative Modelle auch ohne klassischen Programmieraufwand untersuchen lassen. Computeralgebra bedeutet, daß das Programm in der Regel symbolisch rechnet. Numerische Algorithmen werden nur dann verwendet, wenn die symbolische Berechnung in einem konkreten Fall nicht ausfuihrbar oder zu aufwendig ist. Zusätzlich ist es oft möglich, mit einem Computeralgebra-Programmsystem Lösungen in allgemeiner Form anzugeben bzw. die gewünschte Genauigkeit einer Lösung konkret vorzugeben. „Mathematica“ ist ein ComputeralgebraSystem, das an der TFH Wildau in Forschung und Lehre eingesetzt wird. Wir verwenden es neben Internettexten in HTML-Dateien in unserer Modell- und Beispielsammlung PROMA. PROMA steht für „Projekt Mathematica mit Anwendungsbeispielen aus den Wirtschaftswissenschaften". Die folgenden Ausfuihrungen beschreiben, wie die Autoren Mathematica verwendet haben, um die Studenten durch eine Mischung unterschiedlicher Lehrformen in Sinne von Angeboten an die Lernenden zu motivieren. Das Projekt folgt der Auffassung, daß sich die Vorlesungen und Übungen sehr gut durch Computeralgebra in Verbindung mit dem Internet ergänzen lassen.

In Kapitel 2 wird das verwendete Computeralgebra-System beschrieben. Kapitel 3 stellt das didaktische Kon- zept allgemein und an einem Beispiel dar. Kapitel 4 beinhaltet verschiedene Perspektiven und Entwicklungsmöglichkeiten im Rahmen des hier vorgestellten Projektes.

\section{Mathematica}

\section{1 Überblick}

Das Programmsystem Mathematica zeigt mit dem Untertitel „A System For Doing Mathematics By Computer" die vom Autor wohl bedachte Anwendungsrichtung. Es wird von der Firma Wolfram Research Inc, Champaign Illinois, USA seit Januar 1989 für PC's vertrieben, ist inzwischen weltweit verbreitet, wird für eine große Zahl unterschiedlichster Computer-Plattformen angeboten und ist zur Zeit in der Version 3.0.2 erhältlich. Der Architekt ist der Firmengründer Professor Stephen Wolfram selbst, der an der Universität von Illinois als Professor für Physik, Mathematik und Informatik das Center for Complex Systems Research leitete. Von ihm stammt auch das exzellente Benutzerhandbuch [5]. Folgende Anwendungen, die vor allem in Lehre, Forschung und Entwicklung eine große Bedeutung besitzen, werden mit Mathematica PC-gestuitzt nutzbar:

- symbolisches Rechnen (Computeralgebra-System und Modellierungsumgebung)

wie Bruchrechnung, Rechnen mit komplexen Zahlen, Vektor- und Matrixrechnungen, Rechnungen mit Polynomen, Vereinfachung von rationalen Ausdrücken, Grenzwertberechnungen, Reihenentwicklungen, symbolisches Differenzieren und Integrieren, Kurvenanpassungen, Lösen von Gleichungen und Gleichungssystemen, Lösen von Anfangswertproblemen gewöhnlicher Differentialgleichungen

- numerische Auswertungen (numerischer Modellauswerter)

in allen beim symbolischen Rechnen aufgefuihrten Fällen

- Funktions- und Flächengraphendarstellungen in 2D und 3D (Visualisierungssystem)

- Listenverarbeitungen (Listenmanager u.a. für Matrix- und Tabellenarbeit)

z.B. mit Meßreihendaten, Tabellen- und Matrixwerten

- funktionale/prozedurale/regelbasierte Programmierung (Entwicklungsumgebung)

mit der Mathematica-Programmentwicklungsumgebung und Bedienoberfläche Mathematica-Notebook für verschiedene Computer-Betriebssysteme. Der einfachste Einsatz von Mathematica ist der als Taschenrechner mit über 750 eingebauten Operationen und einer umfangreichen Bibliothek an Funktionen. Zur 
Verfuigung stehen a) mehrere Algorithmenbibliotheken („Standard Mathematica Packages“), b) eine stark anwachsende Zahl von kompletten Anwendungsgebietspaketen („Mathematica Applications Library“) - letztere z.B. als „Experimental Data Analyst“, „Finance Essentials" oder „Time Series Pack“ - und c) sogenannte Third-Party Applications z.B. mit den Paketen „Cool Oeconomics Pack“ oder „Global Optimization“. Dadurch und durch weitere Möglichkeiten ist Mathematica ein offenes Softwaresystem, das auf vielfältige Weise individuell angepaßt werden werden kann.

Hinzu kommen eine ganze Reihe von Besonderheiten, die Mathematica so erfolgreich haben werden lassen: grundlegender Einsatz von Transformationsregeln, numerische Berechnungen mit beliebiger vom Nutzer festlegbarer Genauigkeit, Darstellung aller MathematicaGraphiken in einem leicht portierbaren Format - einem Standard-PostScript Format-, Erzeugung von Animationsgraphiken, konsequente Nutzung einer regelbasierten Programmierung mit Mustererkennung und Objektorientiertheit, ausgefeilte Möglichkeiten für den Datenaustausch mit anderen Programmsystemen wie C, Fortran, und über MathLink auch mit Word, Excel und anderen Basisanwendungs-Programmsystemen. Mit
MathLink lassen sich auch Programme erstellen, die Mathematica aufrufen, so als wäre es ein Unterprogramm. Umgekehrt können Eingaben und Anweisungen mit der Mathematica-Sprache erstellt werden, um sie via MathLink an externe Programme zu senden.

Das eigentliche Mathematica-Programm, der Kern („Kernel“), wurde in einer eigens für Mathematica entwickelten objektorientierten Erweiterung der Programmiersprache $C$ geschrieben. Durch ein Vorübersetzungsprogramm kann aus einem Mathematica-Programm ein C-Code erzeugt und auf verschiedenen Zielsystemen in ein ausfuihrbares Programm übersetzt werden. Die neben dem Kern vorhandene Mathematica-Benutzeroberfläche („Mathematica“) ist für den Nutzerdialog zuständig. Mit ihr werden nicht nur - wie sonst in Anwenderprogrammsystemen ïblich - einzelne Nutzeraktionen ausgefuihrt. Es werden mit ihr in einer Art Ablaufprotokoll (Mathematica-Programm oder Mathematica-Notebook) Objekte erstellt, die zugleich die MathematicaSpeichereinheiten darstellen. Sie heißen Notebooks und führen die Dateinamenerweiterung „nb“. Inzwischen können mit Mathematica auch internettaugliche HTMLDateien erzeugt werden. Dies kann und soll hier wesentlich ausgenutzt werden.

\section{gFROMA 1111.nb}

\section{Modell}

Parameter

B Bedarf [1]E]

cF bestell fixe Kosten einer Bestellung [GE]

cL Lagerkosten pro Yengeneinheit [GE / IE]

Variabien

x Bestellmentge [1E], $\mathrm{x} \approx(0, \infty)$

Gleichung

$$
\begin{aligned}
& \mathrm{K}\left[\mathrm{B}, \mathrm{CF}, \mathrm{CL}, \mathrm{x}_{-}\right]=\mathrm{CE} \mathrm{B} / \mathrm{x}+\mathrm{CL} \mathrm{x} \\
& \frac{\mathrm{BCF}}{\mathrm{x}}+\mathrm{cL} \mathrm{x}
\end{aligned}
$$

\section{Lösung in geschlossener Form}

$$
\begin{aligned}
& \mathrm{K}^{\prime}\left[\mathrm{B}_{-}, \mathrm{CF}_{-}, \mathrm{CL}_{-}, \mathrm{x}_{-}\right]=\mathrm{D}[\mathrm{K}[\mathrm{B}, \mathrm{cF}, \mathrm{CL}, \mathrm{x}], \mathrm{x}] \\
& \mathrm{CL}-\frac{\mathrm{BcF}}{\mathrm{x}^{2}}
\end{aligned}
$$

Replace $\left[x, \operatorname{Last}\left[\right.\right.$ Solve $\left.\left.\left[K^{\prime}[p B, p c F, p c L, x]==0, x\right]\right]\right]$

$$
\begin{aligned}
& \frac{\sqrt{\mathrm{pB}} \sqrt{\mathrm{pcF}}}{\sqrt{\mathrm{pcL}}} \\
& \left.\mathrm{f}_{-}, \mathrm{cF}_{-}, \mathrm{cL}\right]=\{\mathrm{pB}=\mathrm{B} ; \mathrm{pcF}=\mathrm{cF} ; \mathrm{pcL}=\mathrm{CL} ; \mathrm{s}\} \\
& \left\{\frac{\sqrt{\mathrm{B}} \sqrt{\mathrm{cF}}}{\sqrt{\mathrm{CL}}}\right\}
\end{aligned}
$$


Als Beispiel (siehe Abb. 1) diene hier ein Ausschnitt aus einem Mathematica-Notebook mit der Modellbeschreibung des hier betrachteten statischen Grundmodells der Bestellmengenplanung (Modul 1111) nach Harris bzw. Andler (siehe [4], [6]).

\subsection{Nutzung von Mathematica für die Lehre}

Die Nutzung von Mathematica in der Lehre hat sich inzwischen weltweit durchgesetzt durch die große Vielzahl und Vielfalt überzeugender Lösungen. Es wird daher inzwischen auch in speziellen Versionen wie "Mathematica Classroom Packs“, „Mathematica For Students“ und „Mathematica Teacher's Edition“ angeboten. Inzwischen bündelt Wolfram Research viele Lehraktivtäten in „Grant Programs for Teaching Enrichment“ sowie in einer „MathSource“ genannten und im Internet angebotenen elektronischen Datenbank für online-Anregungen und Ideen (www.mathsource.com). Viele Mathematica-Anwendungen in der Lehre werden auf den „Annual Mathematica Conferences“ oder in der vierteljährlich erscheinenden Zeitschrift „Mathematica In Education" vorgestellt.

Der Mathematica-Einsatz in der Lehre gründet sich vor allem in der Möglichkeit, durch die spezielle programmartige Mathematica-Notation alle in einer Vorlesung vorzufuihrenden Texte, Bilder und vor allem Formeln als Mathematica-Notebooks zusammenzustellen und während des Vortrages schrittweise zu aktivieren. Einzelne Passagen können auch zunächst unsichtbar sein, um die Übersicht nicht zu stören und um nach der Aktivierung sichtbar den Fluß der Darstellung zu beleben.

Die von den Autoren mit Mathematica bereitgestellte Modell- und Beispielsammlung PROMA kann so auch von anderen interessierten Kollegen Hochschullehrern benutzt werden. Zusätzlich verfolgen wir das Ziel, daß Studenten diese Anwendungsbeispielsammlung nutzen, um interaktives und prozeßorientiertes Lernen zu praktizieren. Dabei können sie die programmierten mathe- matischen Modellbeispiele mit den angegebenen oder eigenen Parametersätzen aktivieren und eine Ergebnisanalyse durchführen (siehe Abschn. 3.1 und 3.2 sowie Abb. 8).

\section{Didaktisches Konzept}

\subsection{Lernumgebung}

Auf dem Wege in die zu erwartende Wissens- und Dienstleistungsgesellschaft ist wohl ein Übergang von der belehrten in eine lemende Gesellschaft unumgänglich. Es wächst offenbar die Bedeutung von Telelearning und Teleteaching/Teletutoring mit einer wachsenden Verantwortung für Moderation und Betreuung durch die Lehrer. Die Akzeptanz durch die Lerner wird in dieser Entwicklung vor allem durch folgende Aspekte bestimmt:

- hohe Interaktivität mit Prozeßorientierung statt mehr passives Lesen

- Nutzung von Animationen und Simulationen (WasWäre-Wenn-Analysen)

- Einsatz von audiovisuellen Mitteln, vor allem aber von (u.U. auch beweglichen) Schemata, Diagrammen, Graphiken, Photos und Videos

- Zeitsouveränität für zeitlich selbstbestimmtes Lernen

- Individualität für selbstorganisiertes und selbstgesteuertes Lernen.

Neben dieser Sammlung von Fakten, die nicht vollständig ist, wird vor allem unter ganzheitlichen Betrachtungspunkten in der Literatur von der Notwendigkeit der Präsentation einer Lernumgebung gesprochen, in der neben der Darstellung von Informationen, dem Sichern und Fördern von Verstehen, Behalten und dem Fertigkeitenausbilden auch die Sicherung und Steigerung von Einstellungen und Motivationen, eine Aufmerksamkeitssteuerung sowie Kommunikationen und Kooperationen gewährleistet werden sollte. Hier wird vor allem darauf

\begin{tabular}{|l|l|}
\hline BeschreibungsEinheiten & Modul der Beispielsammlung \\
\hline Problembezeichnung & Statisches Grundmodell der Bestellmengenplanung \\
\hline ModulNummer & 111 \\
\hline Schwierigkeitsgrad & 1 \\
\hline Thematische Einordnung & Lagerhaltung \\
\hline verwendete Programmumgebung & Mathematica \\
\hline Methoden & der Analysis \\
\hline Verweise & Siehe auch dynamisches Grundmodell der Bestellmengenplanung \\
\hline Kurzbeschreibung & $\ldots$ \\
\hline Problemstellung & $\ldots$ \\
\hline $\begin{array}{l}\text { Modell } \\
\text { - Indizes } \\
\text { - Parameter }\end{array}$ & $\ldots$ \\
\hline - Variablen & \\
\hline Gleichungen & \\
\hline Lösung in geschlossener Form & \\
\hline Ergebnisauswertung & $\ldots$ \\
\hline Beispielanwendung & $\ldots$ \\
\hline Anmerkungen & $\ldots$ \\
\hline Literatur & $\ldots$ \\
\hline
\end{tabular}


verwiesen, daß es neben der didaktischen, der informationstechnischen und der sachlichinhaltlichen Lernumwelt auch soziokulturelle Rahmenbedingungen zu beachten gilt, die vom Lerner als Umgebung mit Atmosphäre empfunden werden kann (siehe [2]).

Wir wollen mit der Bereitstellung und dem Nutzungsangebot einer durch Mathematica informationstechnisch und durch unsere Sammlung mit Anwendungsbeispielen aus den Wirtschaftswissenschaften substanzwissenschaftlich geprägten Lernumgebung vor allem die Interaktivität, den Experimentiercharakter, die Prozeßorientierung und die Anpassungsfähigkeit des Lernvorganges bei selbstbestimmtem und selbstorganisietem Lernen unterstiitzen.

Aus diesem Grund sind alle behandelten Themen unserer Anwendungsbeispielsammlung einzeln als Modul eingerichtet, das jeweils nach dem in Abb. 2 dargestellten einheitlichen Schema aufgebaut wurde:

Die Auflistung dieser Gliederungspunkte gibt zugleich eine grobe Navigationsstrategie für das Bearbeiten im Top-Down-Konzept wieder. Neben den einfuihrenden und erläuternden Mathematica-Textformen in eigenen Abschnitten, den sogenannten Mathematica-NotebookZellen, gibt es dazwischen die entsprechenden Variablenwertebelegungen, Formeln und Mathematica-Befehle in editierbaren und aktivierbaren einzeilig geschriebenen Input-Zellen (mit IN|n]:= gekennzeichnet und uiber n durchnumeriert). Die letzteren können auf Wunsch ausgefuihrt werden, wobei Mathematica unmittelbar im Anschluß die Auswertungsergebnisse in nicht veränderbaren mehrzeilig angeordneten Output-Zellen (mit Out[n]= gekennzeichnet und ebenfalls über $\mathrm{n}$ durchnumeriert) ausgibt. (siehe Abb. 1, 8).

Dabei können auch Wiederholungen durch Zurïckblättern im Mathematica-Notebook und das Aktivieren einer früheren Input-Zelle realisiert werden. Die für strukturierte Programmierung uiblichen Programmsteuerstrukturen für Wiederholungen, Verzweigungen und Unterprogrammorganisation sind in der Mathematica-Programmiersprache auch vorhanden und als Funktionen wie z.B. For [ start, test, inkrement, rumpf ] und

If | bedingung, true, false | anwendbar und aufrufbar. Der Lernende wird mit Texten durch den Modellbeispielerklärungsteil und den Modellbeispielausführungsalgorithmus geführt. Er muß aber nicht der angebotenen Standardnavigation folgen, sondern kann - insbesondere bei wiederholter Nutzung des Beispielmoduls - eine eigene Navigations- und Lösungsstrategie entwikkeln und verfolgen.

Das entscheidende Augenmerk legen die Autoren darauf, daß die Studenten am Beispielmodell komplexe Zusammenhänge begreifen und sich die Art der Abhängigkeiten von Ergebniswerten von einzelnen Eingangsparametern durch Experimente erarbeiten. Die online gefuihrte Auswertung der mathematischen Modelle der Betriebswirtschaftslehre steht also im Mittelpunkt der Nutzung der Anwendungsbeispielsammlung PROMA. Mit solchen Was-Wäre-Wenn-Rechnungen und Simulationen können die Lernenden zugleich Einsichten für eine Anwendungs-, Sensitivitäts- und Stabilitätsanalyse sammeln. Schließlich sollen sie durch diese in Moduln zusam- mengefaßten Modellbeispiele auch angeregt werden, die mathematischen Modelle selbst durch einfache Manipulationen zu verändern und gegebenfalls weiterzuentwickeln.

\subsection{Lernformen}

Im Rahmen dieses Projektes wird ein didaktisches Konzept verfolgt, das auf die möglichst effektive Nutzung aller vorhandenen Ressourcen abzielt. Die finanziellen Mittel zur Beschaffung von Hardware und Software sind knapp. Daher sollten sich die Studenten einen Teil des Lernstoffs am eigenen Schreibtisch, u.U. am eigenen PC und an hochschuleigenen PCs erarbeiten. Sie bringen dann bei der Benutzung der Software in den HochschulLaboren, auf deren PCs neben einem Internet-BrowserProgramm auch das Mathematica-Programmsystem sowie PROMA installiert sind, das nötige Basiswissen mit. In den Laboren kann dann sofort mit der eigenständigen Lösung der Aufgaben begonnen werden. Ziel ist es, den Studenten für jede Aufgabenstellung folgendes Lehrmaterial zur Verfuigung zu stellen:

- HTML-Dateien mit einführenden Beispielen und den notwendigen Erläuterungen in übersichtlicher Form

- Mathematica-Programme mit Kurzdokumentationen der wichtigsten Bedienungselemente und Funktionen von Mathematica

- PROMA als Programmbibliothek mit vorbereiteten Modellen und Anwendungsbeispielen sowie ausgewählten Parametersätzen und zugehörigen Lösungsdatensätzen z.B. aus den Bereichen Lagerhaltung, Produktion, Distribution und Warteschlangentheorie.

Es sind drei verschiedene Lernformen zu unterscheiden:

- Lesen der HTML-Dateien (Phase 1)

- Lesen der Unterlagen, die in Papier- oder Buchform vorliegen (Phase 2)

- Arbeiten mit einem Browser zu Hause oder mit Mathematica bzw. einem Browser im Labor (Phase 3). Der Student oder die Studentin beginnen in der Regel mit dem Arbeiten in einer HTML-Datei. Es bieten sich zwei Möglichkeiten an: (a) Die HTML-Datei wird den Studenten auf Diskette oder CD ROM übergeben. Sie kann dann an einem PC mit einem geeigneten Browser oder z.B. mit MS Word geöffnet und im sogenannten Offline-Modus (ohne Datenuibertragung) benutzt werden. (b) Die Studenten können auf eine HTML-Datei zugreifen, die im Internet zur Verfuigung gestellt wird (Online-Modus). Die zweite Möglichkeit hat den Vorteil, daß sich das Lehrmaterial leichter pflegen läßt. Bei der ersten Nutzungsmöglichkeit entfallen aber die Verbindungskosten. Der Hauptbestandteil des Lehrmaterials sind einfuihrende und erläuternde Texte, Mathematica-Programme, Graphiken und Ergebnisausdrucke. Mit Hilfe der als HTML-Dateien angebotenen Referenztexte können sich die Nutzer einen Überblick über die Anwendungsmöglichkeiten des jeweiligen Modells und seiner Auswertung mit einem MathematicaProgramm verschaffen. Sie entscheiden, ob sie sich mehr Zeit für Mathematica nehmen wollen. Die umfangreiche Primär- und Sekundärliteratur zu Mathematica wirkt auf den Neuling tendenziell abschreckend. Daher sind in Phase 2 die Unterlagen zu lesen, die die konkret zu benutzenden Bedienungselemente und Funktionen von Mathematica als Hostprogramm erklären. 


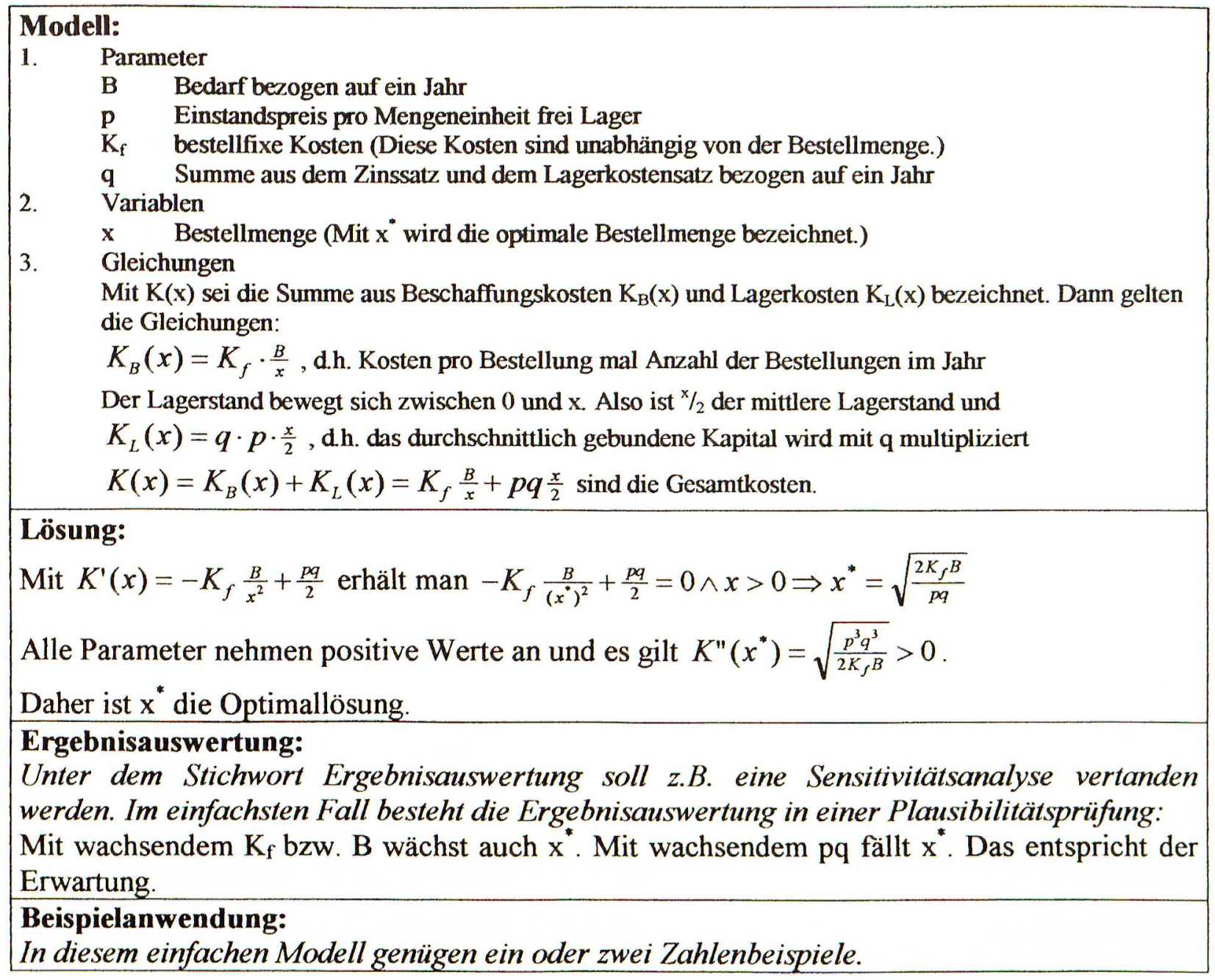

Abb. 3

Das Projektziel besteht nicht darin, das gesamte Lernmaterial auf den PC zu bringen. Bewußt sollen auch Unterlagen in Papierform eingesetzt werden, da reines Arbeiten am Rechner auch ermuidend wirken kann. Wir regen zu multimedialen Arbeitsweisen an. „Multimedial" ist in diesem Sinne zu uibersetzen mit „Einsatz unterschiedlicher Medien wie Text, Tabellen, Graphiken, Farbe, Ton und Video, um Langeweile zu vermeiden“. Die endguiltige Erarbeitung und Festigung der Inhalte erfolgt in Phase 3: Der Lernende nutzt einen der Laborrechner, auf dem Mathematica und PROMA installiert sind. Er muß sich die Modelle jedoch nicht mühsam neu aufbauen, sondern aus den zu den einzelnen Themen vorbereiteten Anwendungsbeispielen auswählen. Die Erfahrung zeigt, daß es sinnvoll ist, dem Lernenden eine existierende Anwendung als Rahmen vorzugeben. Dieser Rahmen kann spielerisch modifiziert werden. Der experimentelle Umgang mit Modellen wird durch die Möglichkeiten von Mathematica unterstiitzt. Ohne großen Aufwand kann der Student Änderungen der Parameter oder der Modellgleichungen vornehmen und deren Auswirkungen beobachten (siehe Abb. 8). Auf diese Weise lernt der Student zum einen die Modelleigenschaften des konkreten Anwendungsbeispieles und die Möglichkeiten und Grenzen seiner Auswertung durch das Mathematica-Programm kennen. Nach den ersten Sitzungen sind die Grundlagen für das Studium der weiterführenden Literatur vorhanden.

\subsection{Lerneinheiten}

Unter einer Lerneinheit verstehen wir im Rahmen von PROMA einen thematisch abgegrenzten Stoff, der in überschaubarer Zeit durchgearbeitet werden kann. Eine Einheit ist z.B. das statische Grundmodell der Bestellmengenoptimierung nach Harris bzw. Andler (siehe Abschnitt 3.4 sowie Abb. 3). Jede Lerneinheit wird in den Dateien und in den ausgedruckten Unterlagen durch eine eindeutige Gliederungsnummer identifiziert. Der Lernende erkennt sofort, welche Unterlagen und Dateien zu dem aktuell bearbeiteten Thema bzw. Themengebiet gehören. Dem Studenten soll der Stoff möglichst transparent gemacht werden. Jede Lerneinheit ist einheitlich nach dem in Abb. 2 ausgewiesenen Schema aufgebaut. Die meisten Einträge der Tabelle sprechen für sich. Folgende Punkte sollen jedoch näher erläutert werden:

- Der Schwierigkeitsgrad gibt an, ob das Problem für Anfänger geeignet ist. Es gibt drei Stufen (1: einfach, 2: mittel, 3: schwer). Der Lernende sollte zuerst die einfachen Probleme aller Themenbereiche durcharbeiten

- Lernenden, die nur an bestimmten Themenbereichen interessiert sind (z.B. an Problemen der Lagerhaltung oder an Aufgaben aus dem Bereich der Distribution), gibt die thematische Einordnung einen Hinweis auf sie interessierende Lerneinheiten

- Der Eintrag fuir die verwendete Software ist notwendig, da später vielleicht auch andere Programme außer Mathematica eingesetzt werden sollen 


\begin{tabular}{|l|}
$\frac{\text { Das klassische Bestellmengenmodell }}{\text { Annahmen }}$ \\
$\frac{\text { Der Verlauf des Lagerbestandes }}{\text { Die Beschaffungskosten }}$ \\
$\frac{\frac{\text { Die Lagerhaltungskosten }}{\text { Die Gesamtkosten }}}{\text { Die Bestellmengenformel }}$ \\
$\frac{\text { Variablenbeschreibung }}{\text { Herleitung der benötigten Ausdrücke }}$ \\
$\frac{\text { Herleitung der optimalen Bestellmenge }}{\text { Herleitung der optimalen Zyklusdauer }}$ \\
$\frac{\text { Die Kosten der optimalen Lösung }}{\text { Beispielrechnungen }}$ \\
Literaturberverzung
\end{tabular}

Abb. 4

- Das Lösen von Optimierungsaufgaben erfordert unterschiedliche Ansätze. Das statische Grundmodell der Bestellmengenoptimierung z.B. läßt sich analytisch lösen. Die Lösung kann in geschlossener Form angegeben werden. Im Bereich der Distributionsplanung spielen lineare und gemischt-ganzzahlige Programme eine wichtige Rolle. Diese lassen sich lediglich numerisch lösen. Der Hinweis auf die verwendeten Methoden erlaubt es dem Lernenden, sich mit Anwendungen bestimmter Verfahren zu beschäftigen. So wird der Bezug zu einzelnen Studienfächern wie Grundlagen der Mathematik I und II und Operations Research hergestellt

- Die Verweise auf verwandte Problemstellungen werden in HTML-Dateien durch entsprechende Links realisiert. Links lassen sich aber auch vorteilhaft einsetzen, um z.B. alle Probleme, die zur Lagerhaltung gehören, auf einer Seite zusammenzustellen

- Die restlichen Einträge folgen der in der Literatur üblichen Gliederung bei der Darstellung quantitativer Modelle. Die durch '...' gekennzeichneten Inhalte sind zu umfangreich fuir die hier abgedruckte Tabelle. In bezug auf das statische Grundmodell der Bestellmengenplanung finden sich diese Inhalte in Abschnitt 3.4

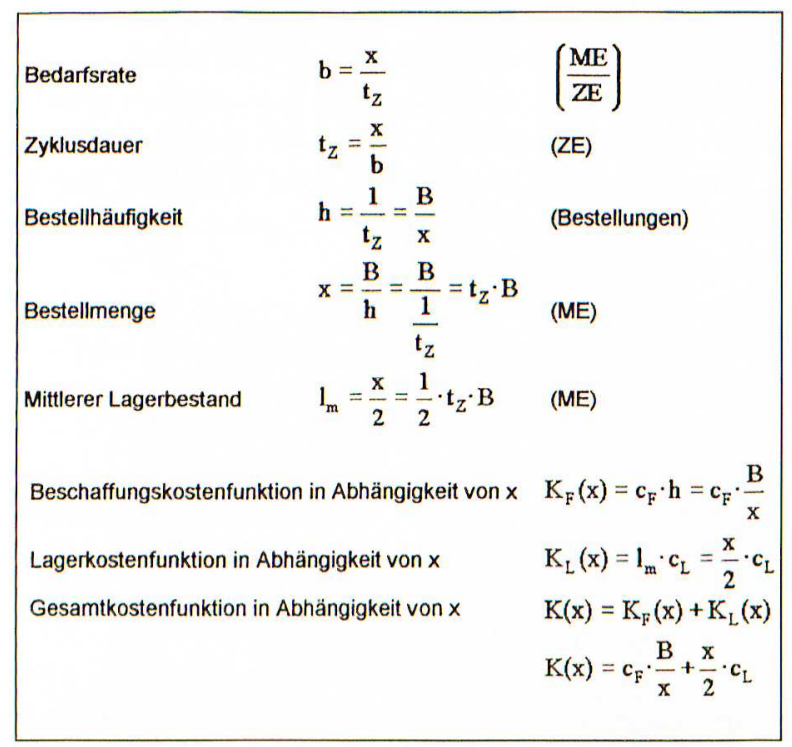

- Unter Ergebnisauswertung verstehen wir hier Sensitivitätsanalysen, postoptimale Analysen usw.

In bezug auf die unter Abschnitt 3.2 angesprochenen Lernformen bedeutet die Gliederung einer Lerneinheit, daß alle zur Verfuigung gestellten HTML-Dateien diesem Aufbau folgen. Das führt zu einer verbesserten Transparenz und hat den Vorteil, daß sich die HTMLDateien und sonstigen Unterlagen leichter pflegen lassen.

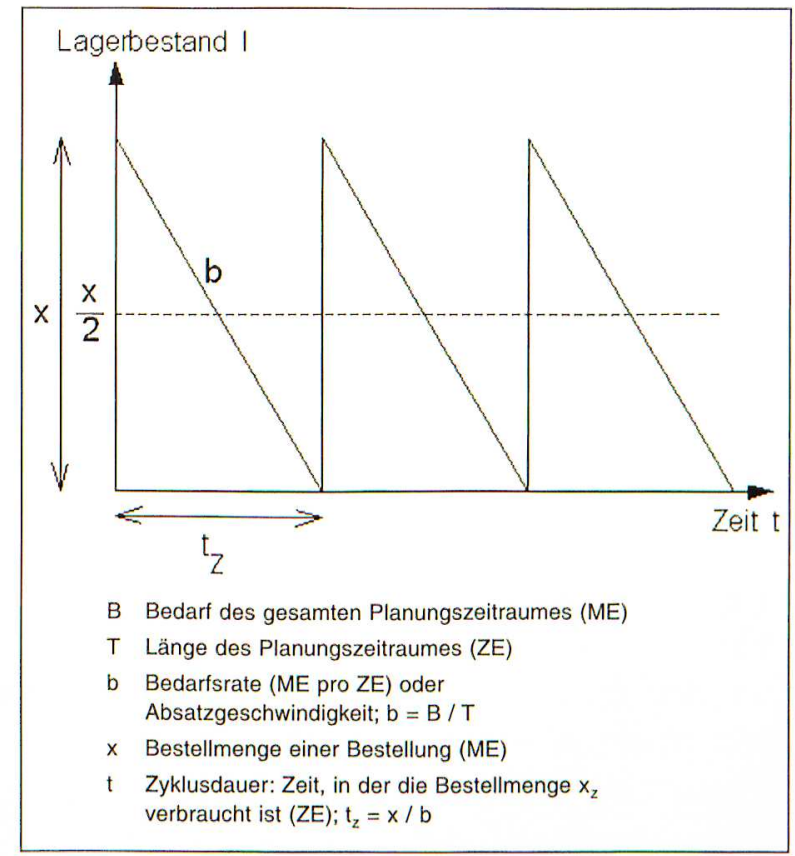

Abb. 6
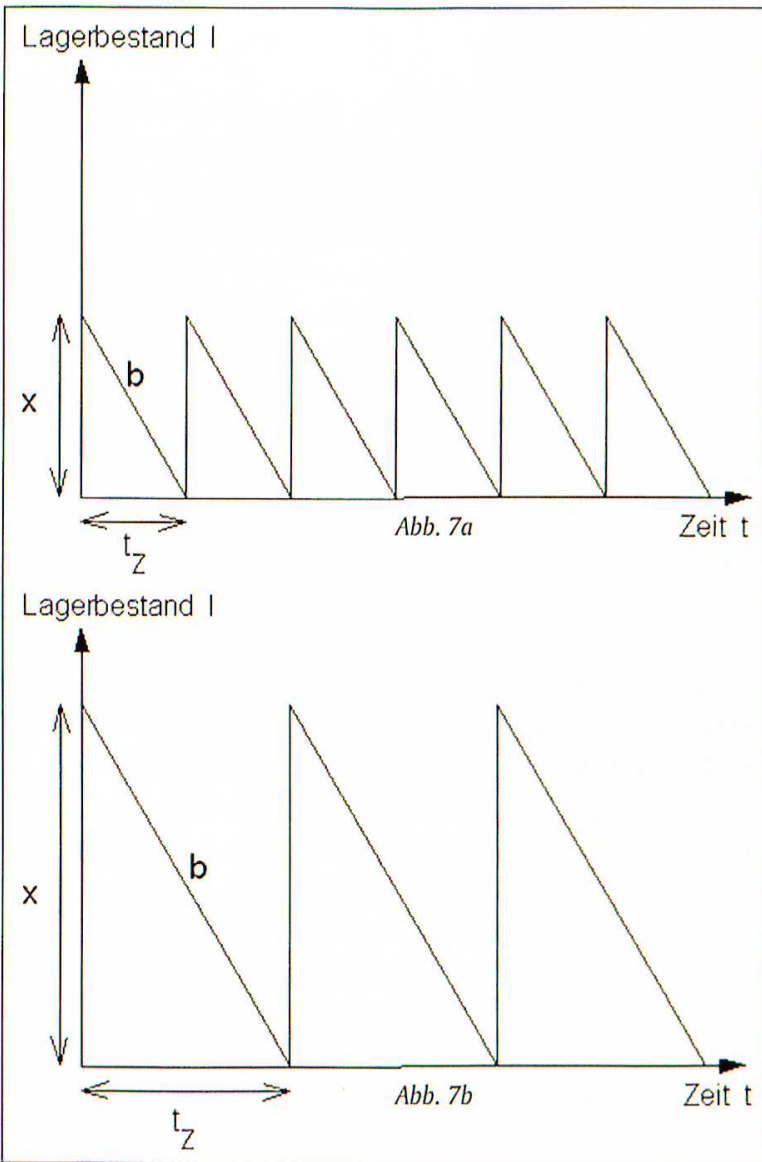

Abb. 7: Bei Erhöhung der Bestellmenge vermindert sich die Anzahl der Bestellungen im Planungszeitraum (siehe Abb. 7a und Abb. 7b) 


\subsection{Beispiel einer Modellformulierung}

Am statischen Grundmodell der Bestellmengenoptimierung (bzw. der Losgrößenplanung) nach Harris bzw. Andler (siehe [4], [6]) läßt sich der Aufbau einer Lerneinheit gut darstellen. Dieses Modell gehört in das Themengebiet Lagerhaltung. Es handelt sich um ein deterministisches Modell. Innerhalb der deterministischen Lagerhaltungsmodelle gehört es zu den statischen Modellen:

\section{Lagerhaltung}

ת,

11 deterministische Lagerhaltungsmodelle त)

111 statische, deterministische Lagerhaltungsmodelle ת)

1111 statisches Grundmodell der Bestellmengenoptimierung

Durch die verwendete Dezimalklassifikation behalten die Nutzer auch bei einer im Laufe der Zeit wachsenden Modellbibliothek den Überblick. Die Tabelle der Abb. 3 zeigt den Aufbau der Lerneinheit für dieses Problem.

\subsection{Training am Modell}

Wie weiter oben schon erläutert, beginnt das Bekanntmachen des Studenten mit dem Anwendungsbeispiel mit einem Modellstudium, das zunächst in HTML-Form über eine CD oder das Internet betrachtet werden kann. Dazu sind alle Texte in markierte Texteinheiten zerlegt, auf die wie im World Wide Web üblich mit Verweisen Vor- oder Zurüickgesprungen werden kann. Die Abb. 4 zeigt die bei dieser Technik übliche Eingangsseite, aufgebaut wie ein Inhaltsverzeichnis und dargestellt als Navigationsleiste. In diesen Texten sind auch Herleitungen der mathematischen Modellgleichungen enthalten, mit denen sich der Student in die Beziehungen der Parameter einlesen und diese stufenweise verarbeiten und aufnehmen kann (siehe Abb. 5).

Neben einfuihrenden Texten und solchen Ableitungen werden hier auch Übersichten und Graphiken angeboten, die Modelleigenschaften deutlich machen und die Studenten befähigen sollen, selbst Modifikationen in den Modellgleichungen anzubringen und deren Auswirkungen durch eine anschließende bildliche Darstellung oder durch Modellauswertungen mit dem Mathematica-Programm zu studieren. Hilfreich sind dazu Graphiken wie sie in den Abb. 6 und Abb. 7 ausschnitthaft gezeigt werden.

Schließlich sollen ein vorbereiteter Beispieltext, das zugehörige Mathematica-Programm mit seinem Quell-

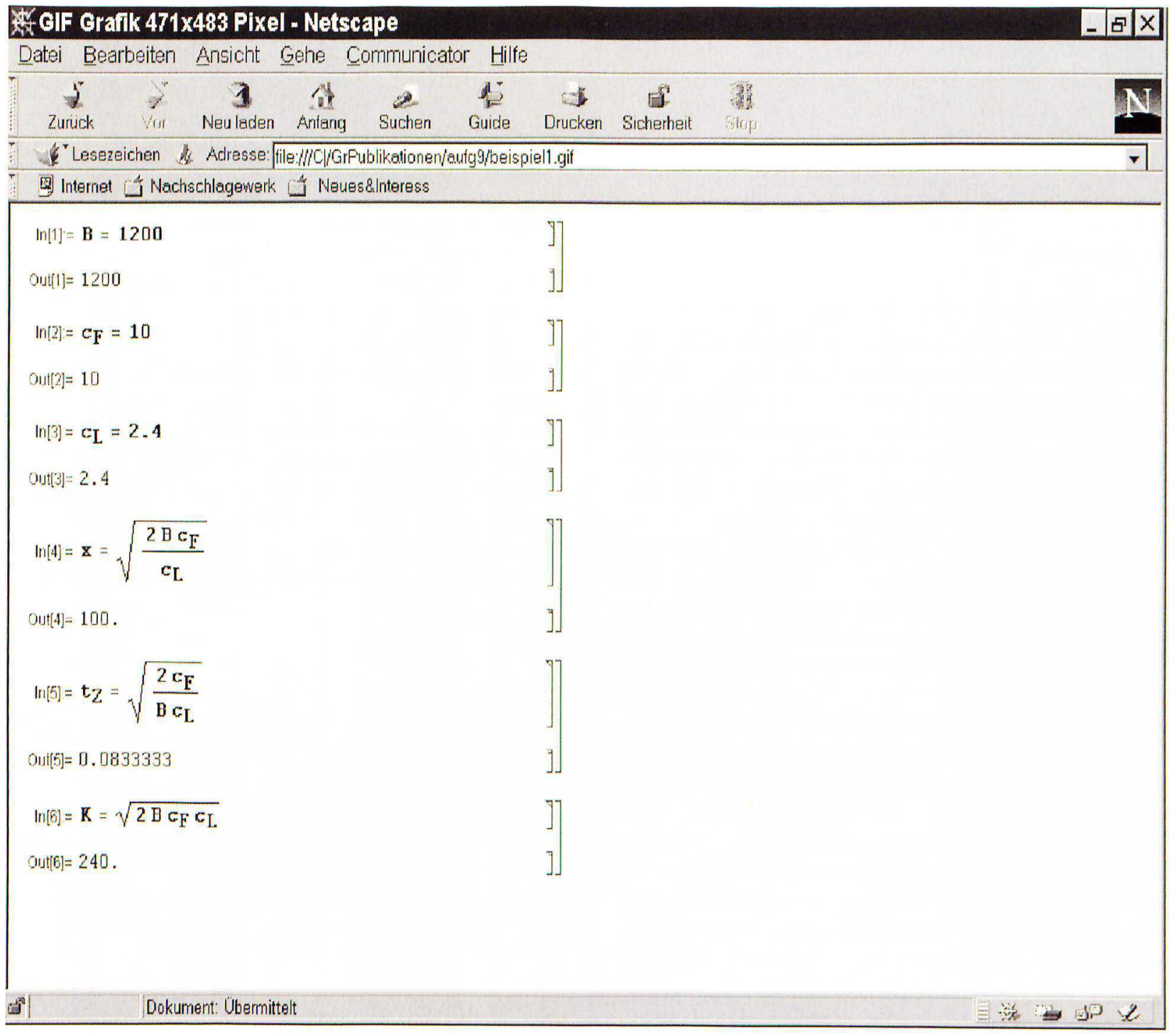


text und die Auswertungsergebnisse in der Abb. 8 die interaktiven Möglichkeiten des Studenten in unserer Lernumgebung verdeutlichen.

Der Mathematica-Aufgabentext, so wie er in einer Mathematica-Zelle steht, hat die folgende Form:

Aufgabenstellung:

In einer Fabrik fällt für ein bestimmtes Einzelteil ein Jahresbedarf von B $=1200$ Stück an. Die bestellfixen Kosten betragen $\mathrm{C}_{\mathrm{F}}=10$,- DM. Die Lagerkosten pro Mengeneinheit betragen $c_{\mathrm{L}}=2,40 \mathrm{DM}$ im Jahr. In welcher Stïckzahl und in welchem zeitlichen Abstand müssen die Einzelteile bestellt werden, um die Gesamtkosten (Bestellkosten + Lagerkosten) so gering wie möglich zu halten, und wie groß sind diese dann?

Lösung:

Diese Aufgabe soll nun mit den uns bekannten Formeln in Mathematica gelöst werden. Die eingegebenen Zeilen kennzeichnet Mathematica mit „In[x]:= , und das Ergebnis dieser Zeilen mit „Out $[x]=$, davor. Mathematicaprogrammquelltext und -ausfuihrungsergebnisse:

(Fortsetzung gemäß Abb. 8)

Erläuterung:

In den ersten drei Eingabezellen wurden die Werte B, $c_{F}$ und $c_{L}$ eingegeben. Anschließend liefert Mathematica nach Eingabe der Formel für die optimale Bestellmenge den Wert 100. Entsprechend erhalten wir nach Formeleingabe für den optimalen Bestellzyklus den Wert 0,0833333 also $1 / 12$ und für die Gesamtkosten den Wert 240.

Um also die minimalen Kosten von 240,- DM zu erreichen muß man jeden Monat 100 Stiick bestellen.

\section{Ausblick}

Der momentane Stand der Arbeiten im Projekt PROMA ist lediglich als ein Ausgangspunkt zu betrachten. Das Projekt ist als konzeptioneller Rahmen angelegt. Es soll möglich sein, eine wachsende Anzahl betriebswirtschaftlicher Modelle und Anwendungsbeispiele in die Bibliothek aufzunehmen. Studenten sollen ermuntert werden, sie interessierende Gebiete im Rahmen von Belegarbeiten einzufuigen. Als ein Beispiel verweisen wir auf den Beleg, den Herr Marko Buchwald (Seminargruppe I 95) im Rahmen der Lehrveranstaltung Produktionswirtschaft $1 \mathrm{im}$ WS 98/99 vorgelegt hat (siehe [1]). Die Abbildungen 3 bis 8 wurden dem PROMA-Modul von Herrn M. Buchwald entnommen (Abbildung 3 in modifizierter Form).

Auf die Existenz von PROMA und die Nutzungsmöglichkeiten der Modell- und Anwendungsbeispielsammlung verweisen wir in unseren Homepages, wo auch über Erarbeitungsfortschritte berichtet werden wird.

Das Projekt soll nicht auf den Einsatz von Mathematica beschränkt sein. Es ist durchaus möglich und sinnvoll, andere Computeralgebra-Programme zu verwenden oder numerische Software einzusetzen. In diesem Falle stellen sich natuirlich einzelne Punkte w.z.B. die Ergebnisauswertung anders dar. Das Prinzip bleibt aber gleich. Eine breitere Verwendung der einzelnen Module könnte durch die Installation von Lehrer-PCs und deren Aus- stattung mit der PROMA-Software in den Unterrichtsräumen erreicht werden.

Leser, die durch diesen Aufsatz ermuntert wurden, sich mit Computeralgebra zu befassen, seien auf den Übersichtsartikel [3] als Einstieg in das Thema verwiesen.

\section{Literatur}

[1] Marko Buchwald, Klassisches Losgrößen-/ Bestellmengenmodell von Harris und Andler, TFH Wildau, FB Betriebswirtschaft/Wirtschaftsinformatik, Belegarbeit 1998, unveröffentlicht

[2] Wolfgang Finke, Raus aus der Lernmisere, Teleworx, Heft 1/98 S44-45, Heft 2/98, S. 38-39

[3] Johannes Grabmeier, Computeralgebra - symbolisches und exaktes Rechnen, Dossier Spektrum der Wissenschaft, Heft 2/99, S. 27-31

[4] Erich Gutenberg, Grundlagen der Betriebswirtschaftslehre, Band 1 [Die Produktion], 24., unveränd. Aufl., Berlin usw. 1983, S. $199 \mathrm{ff}$.

[5] Stephen Wolfram, Mathematica - Ein System für Mathematik auf dem Computer, 2. Auflage, Bonn usw. (Addison Wesley Verlag [Deutschland]), 1994

[6] Günther Zäpfel, Produktionswirtschaft. Operatives Produktions-Management, 1. Aufl., Berlin usw. 1982, Seite $195 \mathrm{ff}$.

\section{Autoren}

Prof. Dr. rer. pol. Matthias Forster

Technische Fachhochschule Wildau

Fachbereich Betriebswirtschaft / Wirtschaftsinformatik

Bahnhofstraße, 15745 Willdau

Tel. (0 33 75) 508-529

E-Mail: mforster@wi-bw.tfh-wildau.de

\section{Dr. rer. nat. Martin Grabow}

Technische Fachhochschule Wildau

Fachbereich Betriebswirtschaft / Wirtschaftsinformatik

Bahnhofstraße, 15745 Willdau

Tel. (0 33 75) 508-250

E-Mail: mgrabow@wi-bw.tfh-wildau.de 